\title{
Review Article \\ Periodontitis and Rheumatoid Arthritis: The Same Inflammatory Mediators?
}

\author{
Fulvia Ceccarelli $(D),{ }^{1}$ Matteo Saccucci $\left(D,{ }^{2}\right.$ Gabriele Di Carlo ${ }^{(D)},{ }^{2}$ Ramona Lucchetti, ${ }^{1}$ \\ Andrea Pilloni $\left(\mathbb{D},{ }^{2}\right.$ Nicola Pranno $\left(\mathbb{1},{ }^{2}\right.$ Valeria Luzzi $\left(\mathbb{1},{ }^{2}\right.$ Guido Valesini, ${ }^{1}$ \\ and Antonella Polimeni $\mathbb{D}^{2}$ \\ ${ }^{1}$ Department of Internal Medicine and Medical Specialties, Sapienza University of Rome, Rome, Italy \\ ${ }^{2}$ Department of Oral and Maxillo-Facial Sciences, Sapienza University of Rome, Viale Regina Elena 287a, 00161 Rome, Italy \\ Correspondence should be addressed to Matteo Saccucci; matteo.saccucci@uniroma1.it
}

Fulvia Ceccarelli, Matteo Saccucci, and Gabriele Di Carlo contributed equally to this work.

Received 7 December 2018; Accepted 6 February 2019; Published 5 May 2019

Guest Editor: Denisse Bravo

Copyright (C) 2019 Fulvia Ceccarelli et al. This is an open access article distributed under the Creative Commons Attribution License, which permits unrestricted use, distribution, and reproduction in any medium, provided the original work is properly cited.

\begin{abstract}
The strict link between periodontitis (PD) and rheumatoid arthritis (RA) has been widely demonstrated by several studies. PD is significantly more frequent in RA patients in comparison with healthy subjects: this prevalence is higher in individuals at the earliest stages of disease and in seropositive patients. This is probably related to the role of $P$. gingivalis in inducing citrullination and leading to the development of the new antigens. Despite the many studies conducted on this topic, there is very little data available concerning the possibility to use the same biomarkers to evaluate both RA and PD patients. The aim of the review is to summarize this issue. Starting from genetic factors, data from literature demonstrated the association between HLA-DRB1 alleles and PD susceptibility, similar to RA patients; moreover, SE-positive patients showed simultaneously structural damage to the wrist and periodontal sites. Contrasting results are available concerning other genetic polymorphisms. Moreover, the possible role of proinflammatory cytokines, such as TNF and IL6 and autoantibodies, specifically anticyclic citrullinated peptide antibodies, has been examined, suggesting the need to perform further studies to better define this issue.
\end{abstract}

\section{Introduction}

Abundant evidences recorded in literature demonstrated that rheumatoid arthritis (RA) and periodontitis (PD) are frequently associated with each other and share several pathogenic and clinical features [1].

RA is a systemic inflammatory autoimmune disease characterized by chronic inflammation and joint tissue destruction, leading to functional disability. Similarly, PD patients experience chronic inflammatory diseases [1-3]. In particular, PD are dysbiotic conditions characterized by an imbalance between subgingival communities and host immune response. The transition from health to PD is characterized by shifts in the community structure of the com- plete subgingival microbiome [4-6].

PD is currently considered a risk factor for RA: the first link between these two conditions was identified in $P$. gingivalis, a gram-negative anaerobe bacteria characterized by the presence of peptidylarginine deiminase (PAD). This enzyme contributes to RA development by catalyzing citrullination, a posttranslational modification playing a crucial role in the production of anticyclic citrullinated peptide antibodies (ACPA), widely recognized as diagnostic and prognostic biomarkers for RA patients [7, 8]. Recently, our group observed a significant association between the percentages of $P$. gingivalis, assessed by real-time PCR, on the total tongue biofilm and RA disease activity (evaluated as disease activity score on 28 joints-DAS28) [9]. This result suggests 
that the oral cavity microbiological status could play a role in the pathogenic mechanisms of inflammation, leading to more active diseases $[9,10]$.

More recently, the role of Aggregatibacter actinomycetemcomitans has been suggested. This oral pathogen could induce hypercitrullination at neutrophil level by secreting leukotoxin A that is able to change neutrophil morphology, mimicking extracellular trap formation. Finally, this process results in the hypercitrullinated autoantigen release, triggering autoimmune response in RA patients [11]. From an epidemiological point of view, several studies have been conducted which remarked the association between RA and PD. The main data is summarized in Table 1. Specifically, case-control studies underline a higher prevalence of $\mathrm{PD}$ in RA patients in comparison with healthy controls [12-24]. PD prevalence is higher in early RA patients at disease onset, despite the young age and paucity of smoking history [14-18]. In addition to higher prevalence, RA patients show a more aggressive PD compared to HS (healthy subjects) $[15,16,18-21]$. Furthermore, some studies have carried out a comparison with OA patients. Dissick and his colleagues observed that PD was more common and severe in RA patients compared to OA. Moreover, in RA, the presence of PD was significantly associated with positivity for rheumatoid factor (RF) and ACPA [13]. More recently, Gonzalez and coauthors observed that ACPApositive RA patients showed significantly higher mean percentage of sites with alveolar bone loss (ABL) greater than $20 \%$ in comparison with OA patients. Moreover, ABL substantially connected to ACPA titers and disease activity in terms of DAS28 [25]. The same cohort was evaluated in terms of HLA-DRB1 and anti-P. gingivalis antibodies, showing higher ACPA levels in patients with subgingival $P$. gingivalis and in those with higher anti-P. gingivalis antibody levels [26].

Moving passed the strict association between RA and PD, from an epidemiological and pathogenic point of view, it is possible to hypothesize a common genetic background and the sharing of inflammatory mediators between RA and PD. The review thoroughly covers this topic.

1.1. Genetic Biomarkers. Several studies have confirmed the role of genetic factors in the RA development: according to a multifactorial model, the interaction between genetic background and environmental factors leads to the development of an autoimmune inflammatory condition, resulting in autoantibodies production. The highly polymorphic HLADRB1 locus (the so-called shared epitope-SE) represents the strongest genetic factor involved in disease development. Particularly, all HLA-DRB alleles with the SE provide RAprone antigen recognition: this leads not only to an increased risk of developing RA but also to the progression into a more erosive, deforming disease. Furthermore, a gene-environment interaction between smoking and SE genes seems to be crucial in the development of seropositive RA. Nonetheless, the contribution of other genetic polymorphisms on RA susceptibility has been also investigated: among these, SNPs in signal transducer and activator of transcription 4 (STAT4), Fc gamma receptor (FCGR), protein tyrosine phosphatase nonreceptor type 22 (PTPN22), PADI-4, tumor necrosis factor (TNF), and interleukin 6 (IL6) genes have been associated with disease development in several case-control studies [27]. It is important to note that genetic polymorphisms could be also associated with different disease phenotypes, in terms of radiographic damage progression [28-30]. Moreover, in 2011, we suggested the possible role of TGF- $\beta$ 869C/T and IL6-174G/C polymorphisms in determining erosive damage evaluated by ultrasonographic assessment in a cohort of RA patients [31].

Some of these genetic factors have been also associated with PD susceptibility, reinforcing the hypothesis of common pathogenic mechanism with RA. Specifically, SE positivity has been widely linked to PD development.

In 2006, Marotte and colleagues investigated the presence of an association between bone destruction at the joint and periodontal level in a wide RA cohort. The analysis of 147 subjects-56.5\% of whom with PD-demonstrated a strong association between PD and wrist destruction, assessed by the radiographic Larsen score. Specifically, the authors identified a significant association between SE positivity and bone destruction in wrist and periodontal sites. In fact, $\mathrm{SE}+$ patients showed 2.5 times greater risk of having wrist joint destruction than $\mathrm{SE}$ - $(\mathrm{OR}=2.5)$. In the same way, $\mathrm{SE}+$ patients had a 2.2 times greater risk to have periodontal destruction compared to SE- $(\mathrm{OR}=2.2)$. The comparison between patients with both site destruction and those without any destruction demonstrated the association with SE positivity ( $\mathrm{OR}=3.9)$. This evidence underlines the possible role of $\mathrm{SE}$ in bone destruction at both sites, suggesting a simultaneous action [32].

Data from Marotte and colleagues agreed with a previous study conducted by Bonfil and colleagues in 1999, suggesting the role of SE as a prognostic factor for PD susceptibility [33].

The possible role of SE-coding DRB1 alleles has been recently underlined by Gehlot and colleagues: the authors observed that transgenic SE+ mice, but not SE- mice, spontaneously developed PD, associated with IL17 overexpression and periostin disruption. Moreover, SE-positive mice showed significantly lower mandibular bone volumetric and mineralization parameters, together with increased alveolar bone resorption [34].

In addition to $\mathrm{SE}$, the possible role of other RA-related genetic polymorphism has been investigated to analyze the association with bone destruction at periodontal level [35]. The studies conducted so far did not produce conclusive results, mainly due to small size cohorts (generally less than 100 patients enrolled), leading to a lack of statistical power to properly detect an association. Hereupon, genotype and allele frequencies could widely vary between different ethnic groups and the same genetic variants could play a different role in different populations. Data from literature provides some evidences to support the association between an aggressive PD and SNPs in interleukin 1 beta (IL1 $\beta$ ), interleukin 1 receptor antagonist (IL1RN), FCGR IIIb, vitamin $\mathrm{D}$ receptor (VDR), and Toll-like receptor 4 (TLR4) genes. Moreover, a chronic $\mathrm{PD}$ was associated with polymorphisms in IL1B, IL1RN, IL6, IL10, VDR, CD14, TLR4, and matrix metalloproteinase-1 (MMP1) genes [34]. The low statistical 







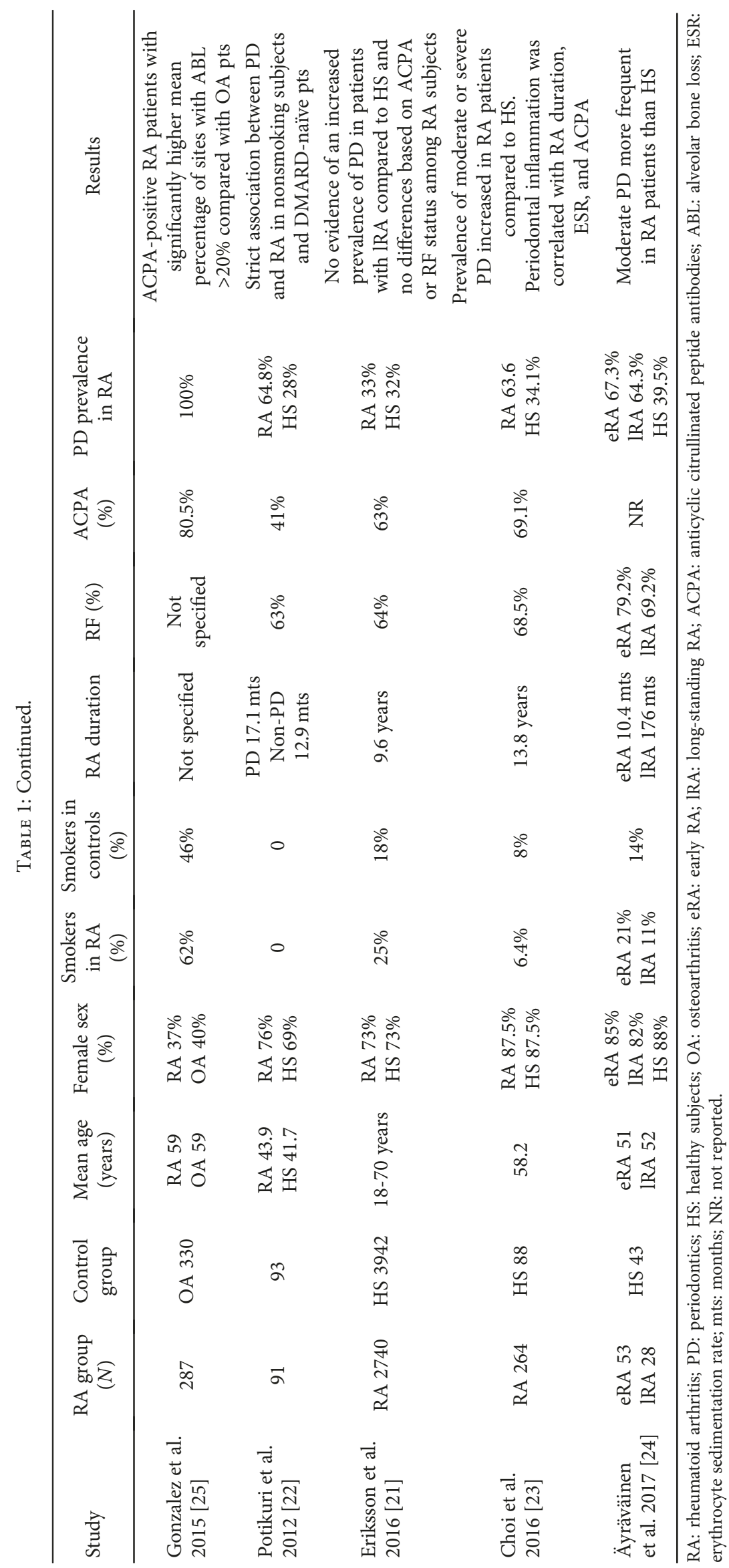


power of these studies was also demonstrated by the results of the meta-analysis conducted by Nikolopoulos and colleagues in 2008, confirming exclusively a moderate and weak positive association between the IL1 composite and IL1B511 genotypes and the occurrence of chronic PD [36]. More recently, the SNP rs2237892 of KCNQ1 gene resulted in a significant association with the coexistence of RA and chronic PD, confirmed in the multiple logistic regression. These results suggest that individuals carrying rs2237892 T allele are likely to have both diseases [37]. The specific role of KCNQ1 gene in RA and PD pathogenesis has not been completely defined: the associated SNP is located in intron 15 of the KCNQ1 gene on chromosome 11p 15.5, encoding the pore-forming $\alpha$ subunit of a voltage-gated $\mathrm{K}^{+}$channel, crucial for the repolarization phase in the cardiac muscle. Moreover, this channel is also expressed at the plasma membrane of fibroblast-like synoviocytes from RA patients and could play a role on cell proliferation and adhesion and secretion of proinflammatory cytokines [38].

1.2. Inflammatory Biomarkers. As widely demonstrated, both RA and PD are characterized by an imbalance between proinflammatory and anti-inflammatory cytokines. In general, high levels of IL1, IL6, and TNF have been demonstrated both in patients with RA and PD. This increased expression of proinflammatory cytokines could stimulate STAT3 activation, playing a key role in the pathophysiology of RA and PD [39].

Particularly, an increased expression of IL1 and TNF has been demonstrated in RA synovium and PD gingival tissues [40]. The central role of inflammatory cytokines in RA pathogenesis has been confirmed by the introduction of biological drugs more than 20 years ago. These drugs are characterized by an innovative mechanism of action, based on the targeted inhibition of specific molecular or cellular targets directly involved in the disease pathogenesis: proinflammatory cytokines (TNF, IL1, and IL6), CTLA-4, and molecules involved in the activation, differentiation, and maturation of $\mathrm{B}$ cells. Their use is associated with better prognosis and the possibility to obtain a clinical remission [41].

Moving on a PD scenario, increased IL1 and TNF levels in periapical exudates were identified in these patients [42]. Moreover, PD progression was reduced by IL1 and TNF inhibitors in experimental models: specifically, histomorphometric analysis indicates that IL1 and TNF antagonists significantly reduced the loss of connective tissue attachment by approximately $51 \%$ and the loss of alveolar bone height by almost 91\% [43]. In 2013, Cetinkaya and colleagues aimed to assess whether PD and RA patients share similar proinflammatory and anti-inflammatory cytokine profiles at a serum and gingival crevicular fluid (GCF) level. The study included 17 RA patients, 16 PD patients, and 16 HS. The authors did not obtain consistent results regarding proinflammatory and anti-inflammatory cytokine levels. Specifically, the total amount and GCF concentration of IL1b, IL4, IL10, and TNF were similar in RA and PD patients. However, the authors underlined the possible influence of the treatment in RA patients [44].

The salivary levels of matrix metalloproteinase- 8 (MMP8) and IL1B were also evaluated in RA patients in comparison with PD and HS. The PD group showed significantly higher salivary levels of MMP-8 and IL1B in comparison with other groups; nevertheless, IL1B was the only biomarker significantly higher in RA compared to controls. Interestingly, RA patients treated by anti-TNF showed lower IL1B and TNF levels compared to nontreated patients [45].

Despite these nonconclusive results, some studies suggest that anticytokine treatment could improve PD. In 2016, Kobayashi and colleagues demonstrated a significant reduction of periodontal inflammation (assessed in terms of gingival index, bleeding on probing, and probing depth) in RA patients treated with tocilizumab and TNF inhibitors. As expected, treatment induced also decreased significantly in RA disease activity parameters, including DAS28-CRP, number of tender and swollen joints, and serum levels of ACPA and RF [46]. This treatment relationship was confirmed also by the evidence that nonsurgical treatment for PD seems to be able to improve the RA activity status: growing evidences demonstrated significant improvement of ESR, CRP, and DAS28 during PD treatment in RA patients $[47,48]$.

\section{Autoantibodies}

Some studies have investigated the presence of RA-related autoantibodies in PD patients. Taken together, PD patients demonstrated a high frequency of ACPA compared to controls; moreover, these antibodies showed a significantly higher titer in PD [3, 49].

However, these studies enrolled small populations and differences between patients and controls were not statistically significant. In 2014, De Pablo and colleagues tested sera from 194 patients with and without PD, none of whom with $\mathrm{RA}$, to assess the presence of different antibodies. PD was associated with a normal frequency of ACPA and antimutated citrullinated vimentin (about 1\%), but a significantly higher frequency of positive anticitrullinated $\alpha$-enolase peptide-1 (anti-CEP-1; 12\% versus $3 \%$ ) and its uncitrullinated form $(16 \%$ versus $2 \%$; $p<0.001)$. Moreover, positive antibodies against uncitrullinated fibrinogen and uncitrullinated equivalent of CCP were more common in PD compared to non-PD patients (26\% versus 3\%; $9 \%$ versus $3 \%$ ). The presence of these autoantibodies was not associated with smoking status, confirming that the PD autoantibody response was not exclusively due to smoking [50]. Moreover, the study conducted by Gonzales in 2015 underlines the possible role of ACPA, observing that ACPA-positive RA patients had a significantly higher mean percentage of sites with ABL greater than $20 \%$ compared to OA controls [51]. More recently, the presence of anticitrullinated histone H3 autoantibodies was investigated: these biomarkers were found in $39 \%$ of RA patients compared to $8 \%$ in $\mathrm{HC}$ and $10 \%$ in PD patients. No associations were found between anticitrullinated histone $\mathrm{H} 3$ levels and periodontal status in RA patients [52].

\section{Conclusions}

Despite the widely demonstrated connection between RA and PD from an epidemiological and pathogenetic point of 


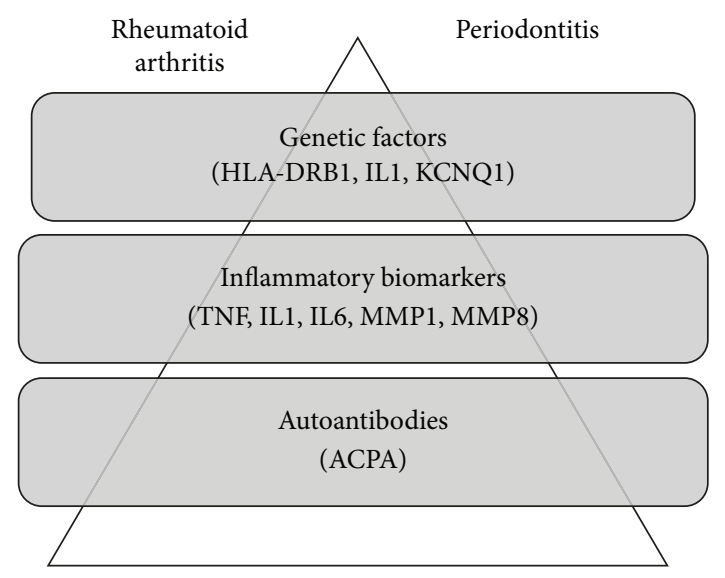

FIGURE 1: Suggested genetic factors, inflammatory biomarkers, and autoantibodies shared by rheumatoid arthritis and periodontitis. IL1: interleukin-1; TNF: tumor necrosis factor; MMP1: matrix metalloproteinase-1; MMP8: matrix metalloproteinase-8; ACPA: anticyclic citrullinated peptide antibodies.

view, data from literature does not seem to support the sharing of the same mediators. Regarding genetic factors, the most consistent data are related to HLA-DRB1 alleles: especially the presence of SE is associated with susceptibility and severity in both diseases. Conversely, contrasting results are available concerning other genetic polymorphisms or proinflammatory cytokines such as TNF, IL1, and IL6. Finally, there are few cases in which RA-related antibodies are found in PD patients. These evidences are graphically represented in Figure 1. Altogether, the paucity of data on this topic suggests that deeper studies, including wider populations and with a longitudinal design, are required to better clarify this issue.

\section{Conflicts of Interest}

The authors declare that there is no conflict of interests regarding the publication of this paper.

\section{Authors' Contributions}

Fulvia Ceccarelli, Matteo Saccucci, and Gabriele Di Carlo contributed equally to this work.

\section{References}

[1] J. Potempa, P. Mydel, and J. Koziel, "The case for periodontitis in the pathogenesis of rheumatoid arthritis," Nature Reviews Rheumatology, vol. 13, no. 10, pp. 606-620, 2017.

[2] P. M. Bartold, R. I. Marshall, and D. R. Haynes, "Periodontitis and rheumatoid arthritis: a review," Journal of Periodontology, vol. 76, no. 11-s, pp. 2066-2074, 2005.

[3] P. De Pablo, I. L. Chapple, C. D. Buckley, and T. Dietrich, "Periodontitis in systemic rheumatic diseases," Nature Reviews Rheumatology, vol. 5, no. 4, pp. 218-224, 2009.

[4] P. I. Diaz, A. Hoare, and B. Y. Hong, "Subgingival microbiome shifts and community dynamics in periodontal diseases," Journal of the California Dental Association, vol. 44, no. 7, pp. 421435, 2016.
[5] R. J. Lamont and G. Hajishengallis, "Polymicrobial synergy and dysbiosis in inflammatory disease," Trends in Molecular Medicine, vol. 21, no. 3, pp. 172-183, 2015.

[6] L. Abusleme, A. K. Dupuy, N. Dutzan et al., "The subgingival microbiome in health and periodontitis and its relationship with community biomass and inflammation," The ISME Journal, vol. 7, no. 5, pp. 1016-1025, 2013.

[7] G. P. Harvey, T. R. Fitzsimmons, A. A. Dhamarpatni, C. Marchant, D. Haynes, and P. Bartold, "Expression of peptidylarginine deiminase- 2 and -4 , citrullinated proteins and anti-citrullinated protein antibodies in human gingiva," Journal of Periodontal Research, vol. 48, no. 2, pp. 252-261, 2013.

[8] G. Valesini, M. C. Gerardi, C. Iannuccelli, V. A. Pacucci, M. Pendolino, and Y. Shoenfeld, "Citrullination and autoimmunity," Autoimmunity Reviews, vol. 14, no. 6, pp. 490-497, 2015.

[9] F. Ceccarelli, G. Orrù, A. Pilloni et al., "Porphyromonas gingivalis in the tongue biofilm is associated with clinical outcome in rheumatoid arthritis patients," Clinical and Experimental Immunology, vol. 194, no. 2, pp. 244-252, 2018.

[10] M. Saccucci, G. Di Carlo, M. Bossù, F. Giovarruscio, A. Salucci, and A. Polimeni, "Autoimmune diseases and their manifestations on oral cavity: diagnosis and clinical management," Journal of Immunology Research, vol. 2018, Article ID 6061825, 6 pages, 2018.

[11] M. F. Konig, L. Abusleme, J. Reinholdt et al., "Aggregatibacter actinomycetemcomitans-induced hypercitrullination links periodontal infection to autoimmunity in rheumatoid arthritis," Science Translational Medicine, vol. 8, no. 369, article 369ra176, 2016.

[12] N. Pischon, T. Pischon, J. Kröger et al., "Association among rheumatoid arthritis, oral hygiene, and periodontitis," Journal of Periodontology, vol. 79, no. 6, pp. 979-986, 2008.

[13] A. Dissick, R. S. Redman, M. Jones et al., "Association of periodontitis with rheumatoid arthritis: a pilot study," Journal of Periodontology, vol. 81, no. 2, pp. 223-230, 2010.

[14] J. U. Scher, C. Ubeda, M. Equinda et al., "Periodontal disease and the oral microbiota in new-onset rheumatoid arthritis," Arthritis \& Rheumatism, vol. 64, no. 10, pp. 3083-3094, 2012.

[15] M. de Smit, J. Westra, A. Vissink, B. D.-v. der Meer, E. Brouwer, and A. van Winkelhoff, "Periodontitis in established rheumatoid arthritis patients: a cross-sectional clinical, microbiological and serological study," Arthritis Research \& Therapy, vol. 14, no. 5, article R222, 2012.

[16] S. B. Ranade and S. Doiphode, "Is there a relationship between periodontitis and rheumatoid arthritis?," Journal of Indian Society of Periodontology, vol. 16, no. 1, pp. 2227, 2012.

[17] S. Reichert, M. Haffner, G. Keyßer et al., "Detection of oral bacterial DNA in synovial fluid," Journal of Clinical Periodontology, vol. 40, no. 6, pp. 591-598, 2013.

[18] B. Wolff, T. Berger, C. Frese et al., "Oral status in patients with early rheumatoid arthritis: a prospective, case-control study," Rheumatology, vol. 53, no. 3, pp. 526-531, 2014.

[19] R. Joseph, S. Rajappan, S. G. Nath, and B. J. Paul, “Association between chronic periodontitis and rheumatoid arthritis: a hospital-based case-control study," Rheumatology International, vol. 33, no. 1, pp. 103-109, 2013.

[20] H. H. Chen, D. Y. Chen, K. L. Lai et al., "Periodontitis and etanercept discontinuation risk in anti-tumor necrosis factornaive rheumatoid arthritis patients: a nationwide population- 
based cohort study," Journal of Clinical Rheumatology, vol. 19, no. 8, pp. 432-438, 2013.

[21] K. Eriksson, L. Nise, A. Kats et al., "Prevalence of periodontitis in patients with established rheumatoid arthritis: a Swedish population based case-control study," PLoS One, vol. 11, no. 5, article e0155956, 2016.

[22] D. Potikuri, K. C. Dannana, S. Kanchinadam et al., "Periodontal disease is significantly higher in non-smoking treatmentnaive rheumatoid arthritis patients: results from a casecontrol study," Annals of the Rheumatic Diseases, vol. 71, no. 9, pp. 1541-1544, 2012.

[23] I. A. Choi, J. H. Kim, Y. M. Kim et al., "Periodontitis is associated with rheumatoid arthritis: a study with longstanding rheumatoid arthritis patients in Korea," The Korean Journal of Internal Medicine, vol. 31, no. 5, pp. 977-986, 2016.

[24] L. Äyräväinen, M. Leirisalo-Repo, A. Kuuliala et al., "Periodontitis in early and chronic rheumatoid arthritis: a prospective follow-up study in Finnish population," BMJ Open, vol. 7, no. 1, article e011916, 2017.

[25] S. M. Gonzalez, J. B. Payne, F. Yu et al., "Alveolar bone loss is associated with circulating anti-citrullinated protein antibody (ACPA) in patients with rheumatoid arthritis," Journal of Periodontology, vol. 86, no. 2, pp. 222-231, 2015.

[26] T. R. Mikuls, J. B. Payne, F. Yu et al., "Periodontitis and Porphyromonas gingivalis in patients with rheumatoid arthritis," Arthritis \& Rhematology, vol. 66, no. 5, pp. 1090-1100, 2014.

[27] C. Perricone, F. Ceccarelli, and G. Valesini, "An overview on the genetic of rheumatoid arthritis: a never-ending story," Autoimmunity Reviews, vol. 10, no. 10, pp. 599-608, 2011.

[28] S. Genevay, F. S. Di Giovine, T. V. Perneger et al., "Association of interleukin- 4 and interleukin-1B gene variants with Larsen score progression in rheumatoid arthritis," Arthritis \& Rheumatism, vol. 47, no. 3, pp. 303-309, 2002.

[29] D. Mewar, I. Marinou, A. L. Coote et al., "Association between radiographic severity of rheumatoid arthritis and shared epitope alleles: differing mechanisms of susceptibility and protection," Annals of the Rheumatic Diseases, vol. 67, no. 7, pp. 980-983, 2008.

[30] J. E. Fonseca, J. Cavaleiro, J. Teles et al., "Contribution for new genetic markers of rheumatoid arthritis activity and severity: sequencing of the tumor necrosis factor-alpha gene promoter," Arthritis Research \& Therapy, vol. 9, no. 2, article R37, 2007.

[31] F. Ceccarelli, C. Perricone, M. Fabris et al., "Transforming growth factor $\beta$ 869C/T and interleukin $6-174 \mathrm{G} / \mathrm{C}$ polymorphisms relate to the severity and progression of bone-erosive damage detected by ultrasound in rheumatoid arthritis," Arthritis Research \& Therapy, vol. 13, no. 4, article R111, 2011.

[32] H. Marotte, P. Farge, P. Gaudin, C. Alexandre, B. Mougin, and P. Miossec, "The association between periodontal disease and joint destruction in rheumatoid arthritis extends the link between the HLA-DR shared epitope and severity of bone destruction," Annals of the Rheumatic Diseases, vol. 65, no. 7, pp. 905-909, 2006.

[33] J. J. Bonfil, F. L. Dillier, P. Mercier et al., "A "case control" study on the rôle of HLA DR4 in severe periodontitis and rapidly progressive periodontitis: identification of types and subtypes using molecular biology (PCR.SSO)," Journal of Clinical Periodontology, vol. 26, no. 2, pp. 77-84, 1999.

[34] P. Gehlot, S. L. Volk, H. F. Rios, K. J. Jepsen, and J. Holoshitz, "Spontaneous destructive periodontitis and skeletal bone damage in transgenic mice carrying a human shared epitope- coding HLA-DRB1 allele," RMD Open, vol. 2, no. 2, article e000349, 2016.

[35] M. L. Laine, W. Crielaard, and B. G. Loos, "Genetic susceptibility to periodontitis," Periodontology 2000, vol. 58, pp. 37-68, 2012.

[36] G. K. Nikolopoulos, N. L. Dimou, S. J. Hamodrakas, and P. G. Bagos, "Cytokine gene polymorphisms in periodontal disease: a meta-analysis of 53 studies including 4178 cases and 4590 controls," Journal of Clinical Periodontology, vol. 35, no. 9, pp. 754-767, 2008.

[37] T. Kobayashi, J. I. Kido, Y. Ishihara et al., “The KCNQ1 gene polymorphism as a shared genetic risk for rheumatoid arthritis and chronic periodontitis in Japanese adults: a pilot casecontrol study," Journal of Periodontology, vol. 89, no. 3, pp. 315-324, 2018.

[38] X. Hu, T. Laragione, L. Sun et al., "KCa1.1 potassium channels regulate key proinflammatory and invasive properties of fibroblast-like synoviocytes in rheumatoid arthritis," Journal of Biological Chemistry, vol. 287, no. 6, pp. 4014-4022, 2012.

[39] T. Kobayashi and H. Yoshie, "Host responses in the link between periodontitis and rheumatoid arthritis," Current Oral Health Reports, vol. 2, no. 1, pp. 1-8, 2015.

[40] C. M. Liu, L. T. Hou, M. Y. Wong, and E. F. Rossomando, "Relationships between clinical parameters, interleukin $1 \mathrm{~B}$ and histopathologic findings of gingival tissue in periodontitis patients," Cytokine, vol. 8, no. 2, pp. 161-167, 1996.

[41] F. Conti, F. Ceccarelli, L. Massaro et al., "Biological therapies in rheumatic diseases," La Clinica Terapeutica, vol. 164, no. 5, pp. e413-e428, 2013.

[42] T. Ataoğlu, M. Ungör, B. Serpek, S. Haliloglu, H. Ataoglu, and H. Ari, "Interleukin-1beta and tumour necrosis factor-alpha levels in periapical exudates," International Endodontic Journal, vol. 35, no. 2, pp. 181-185, 2002.

[43] A. J. Delima, T. Oates, R. Assuma et al., "Soluble antagonists to interleukin-1 (IL-1) and tumor necrosis factor (TNF) inhibits loss of tissue attachment in experimental periodontitis," Journal of Clinical Periodontology, vol. 28, no. 3, pp. 233240, 2001.

[44] B. Cetinkaya, E. Guzeldemir, E. Ogus, and S. Bulut, "Proinflammatory and anti-inflammatory cytokines in gingival crevicular fluid and serum of patients with rheumatoid arthritis and patients with chronic periodontitis," Journal of Periodontology, vol. 84, no. 1, pp. 84-93, 2013.

[45] J. Mirrielees, L. J. Crofford, Y. Lin et al., "Rheumatoid arthritis and salivary biomarkers of periodontal disease," Journal of Clinical Periodontology, vol. 37, no. 12, pp. 1068-1074, 2010.

[46] T. Kobayashi, S. Ito, D. Kobayashi et al., "Serum immunoglobulin G levels to Porphyromonas gingivalis peptidylarginine deiminase affect clinical response to biological disease-modifying antirheumatic drug in rheumatoid arthritis," PLoS One, vol. 11, no. 4, article e0154182, 2016.

[47] G. U. Jung, J. Y. Han, K. G. Hwang, C. J. Park, P. G. Stathopoulou, and J. P. Fiorellini, "Effects of conventional synthetic disease-modifying antirheumatic drugs on response to periodontal treatment in patients with rheumatoid arthritis," BioMed Research International, vol. 2018, Article ID 1465402, 7 pages, 2018.

[48] X. Zhao, Z. Liu, D. Shu et al., "Association of periodontitis with rheumatoid arthritis and the effect of non-surgical periodontal treatment on disease activity in patients with rheumatoid 
arthritis," Medical Science Monitor, vol. 24, pp. 5802-5810, 2018.

[49] N. Wegner, K. Lundberg, A. Kinloch et al., "Autoimmunity to specific citrullinated proteins gives the first clues to the etiology of rheumatoid arthritis," Immunological Reviews, vol. 233, no. 1, pp. 34-54, 2010.

[50] P. De Pablo, T. Dietrich, I. L. C. Chapple et al., "The autoantibody repertoire in periodontitis: a role in the induction of autoimmunity to citrullinated proteins in rheumatoid arthritis?," Annals of the Rheumatic Diseases, vol. 73, no. 3, pp. 580-586, 2014.

[51] J. R. Gonzales, “T- and B-cell subsets in periodontitis," Periodontology 2000, vol. 69, no. 1, pp. 181-200, 2015.

[52] K. M. J. Janssen, M. J. de Smit, C. Withaar et al., “Autoantibodies against citrullinated histone $\mathrm{H} 3$ in rheumatoid arthritis and periodontitis patients," Journal of Clinical Periodontology, vol. 44, no. 6, pp. 577-584, 2017. 


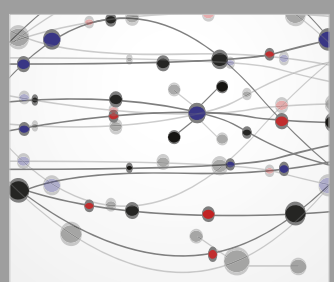

The Scientific World Journal
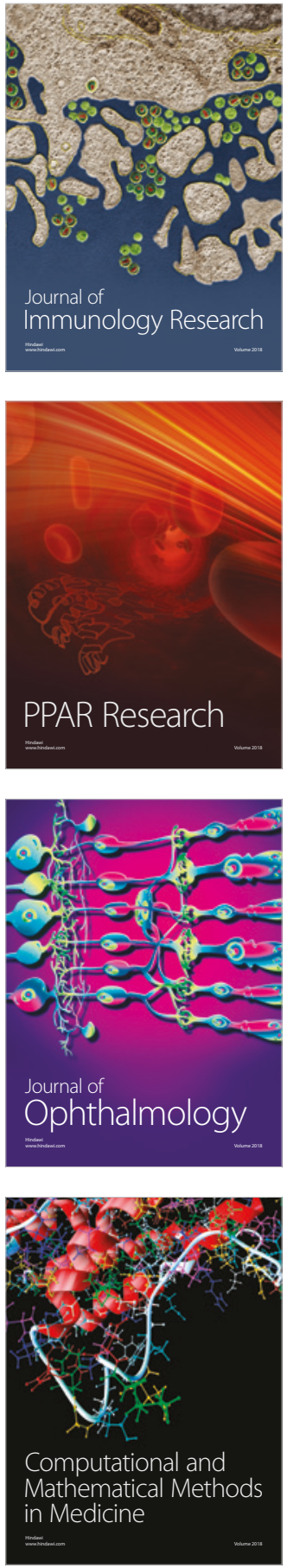

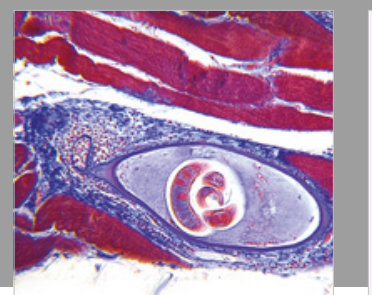

Gastroenterology Research and Practice

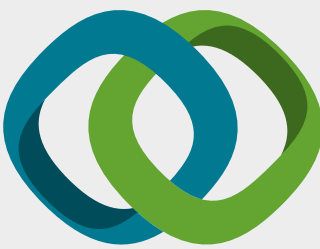

\section{Hindawi}

Submit your manuscripts at

www.hindawi.com
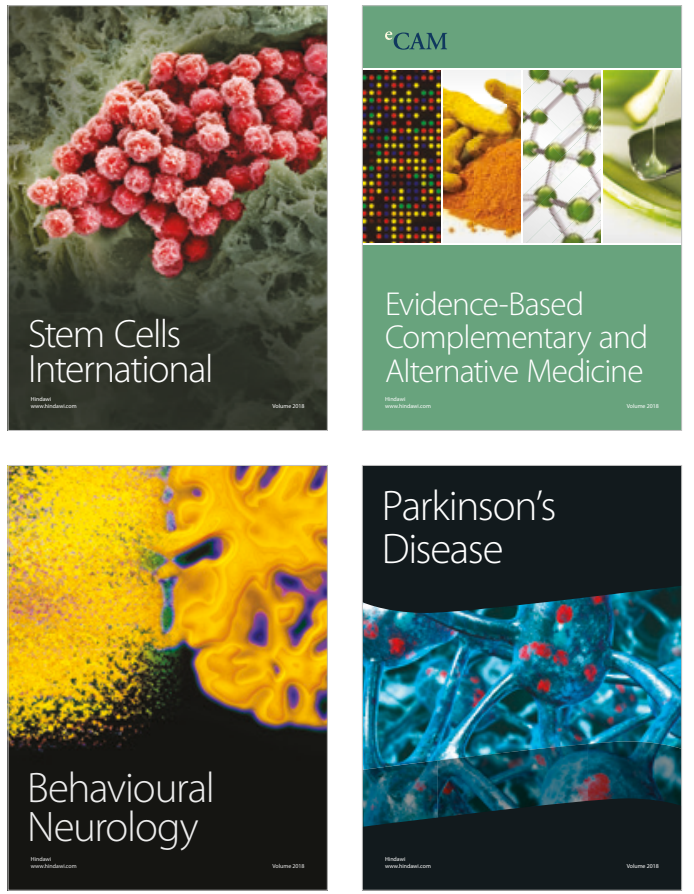

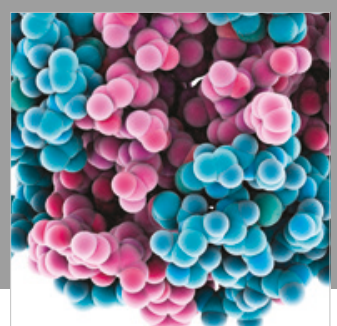

ournal of

Diabetes Research

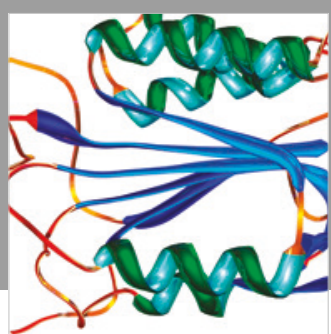

Disease Markers
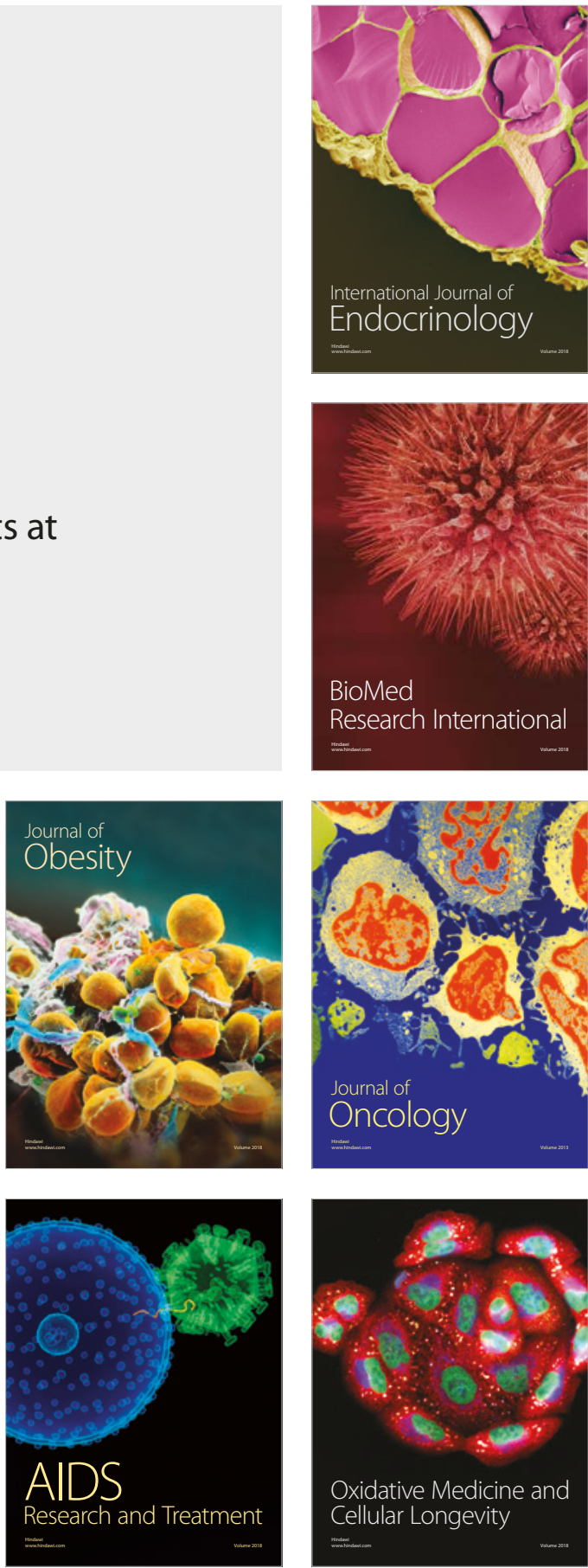